\title{
Uniting Lifecycle Information - From Items to Assets, from Concepts to Practice
}

\author{
Marja Lintala $^{1}$ and Pierre Bonnal ${ }^{2}$ \\ ${ }^{1}$ Karlsruhe Institute of Technology, Institute for Information Management in Engineering, \\ Karlsruhe, Germany \\ ${ }^{2}$ CERN, European Organization for Nuclear Research, Genève, Switzerland \\ marja.lintala@kit.edu, pierre.bonnal@cern.ch
}

\begin{abstract}
Many products have become more and more complex and tailormade. Consequently, product data is an extremely important factor to ensure the success and the sustainability of a product. Large and complex products, systems or facilities were managed in the past without the enhanced information technology systems available nowadays. Among some successful products or systems, one can cite space programs such as the NASA Apollo program or the particle accelerators built as from the 1950s such as that of CERN, Geneva, Switzerland, which serves as a case study for this paper. Some of the products developed in these eras are still in operation. Projects and practitioner approaches were developed and are still under development to address the problem of migrating data, produced with different technologies, to today's technologies. For instance, the ISO 10303 standard-also known as the STEP standard (Standard for the Exchange of Product Data)—provides the means to transfer CAD data between systems. But only little attention has been drawn to providing reliable means for integrating the information from different lifecycle phases together. This paper aims to draw attention to the issues raised by the use of different terminologies and systems in different lifecycle phases, in particular the concept of item during conception and development phase, and the concept of asset during that of operations and maintenance and some problems that this may bring.
\end{abstract}

Keywords: Item, Asset, Product Lifecycle Management, Change Management, CERN.

\section{$1 \quad$ Introduction}

In our constantly changing world, even consumer products have become more and more complex and tailor-made. So it is no wonder that industrial products and systems are increasingly engineered. On the other hand, regulations such as laws, directives and standards are being constantly updated and are becoming stricter and more constraining. For example, in the European Union (EU) and European Economic Area (EEA), Machinery Directive 2006/EC/42 [1] valid as from December 2009 and the related standards dedicate more and more room to safety features. In this quickly evolving environment, product information management throughout the entire lifecycle has become an extremely important factor to ensure the success of a product. 
Just a few decades ago, large and complex products, systems and facilities were conceived, developed, operated and maintained simply with paper archived in filing cabinets. With the advent of microcomputers, files and folders took over. Documents were managed manually and data exchange, in the modern sense, was the manual transportation of documents or later documents in a digital format such as floppy disks [2]. Nowadays, information systems that embed word-processed and CAD data are widely used, especially when the products are complex. Information systems, such as those used in PLM (Product Lifecycle Management), are no longer only technically focused, but take into account the entire product lifecycle and enable the collaboration of various business units from development to service [2].

In products with a long life, and with a great amount of history, information may have been produced with different technologies for different product lifecycle purposes. For instance, the Proton Synchrotron (PS) particle accelerator in CERN (European Organization for Nuclear Research, Geneva, Switzerland) built in the 1950 s is fifty years later still a key component of CERN's accelerator complex. Operations and maintenance of scientific facilities such as that of the PS rely on information originating from various sources: paper documents in filing cabinets, some files still on microcomputers, but also documents in shared access on CERN's several electronic data and document management systems. The in-formation also relates to different lifecycle phases: 2D drawings, engineering specifications, design reports, manufacturing and assembly procedures and records prepared or released in the conception and development phase; and operations and maintenance documentation such as operations and maintenance manuals, procedures and control reports released in the operation and maintaining phase.

Methods for solving the problem of integrating information from different tools, for instance different CAD software, have gained attention over time. Standards such as the ISO 10303 - also known as the STEP standard (Standard for the Exchange of Product Data) [3] — have been developed. Approaches for product architectures, such as modular or integral architecture [4], and for creating a customer demand adaptable product [5], also emerged. However, these methods concentrate on the design phase of the whole product lifecycle, and hence, adding product information from different lifecycle phases needs more attention. The aim of this paper is to draw attention on the issues that arise out of the use of different terminologies and also information systems in different lifecycle and some problems that this may bring.

\section{Lifecycle Management}

The importance of coherency and the integrity of data are widely recognized, because it avoids a loss of time. This 'loss of time' consists of searching and waiting for data, data translations, working with wrong data and recreating the existing knowledge as described in [6]. The Product Lifecycle Management (PLM) approach addresses streamlining the product and related processes, and in-formation flows throughout the product's lifecycle [6]. The aim of PLM is to pro-vide a continuous and transparent flow of digital information within the lifecycle [2]. However, as it is pointed out in [6], the underlying concept in PLM is knowledge management.

Knowledge is according to [7] 'learned information'. Information on the other hand is 'interpreted data' and data 'syntactic entities' [7]. The relationship between 
these three key concepts have been discussed in previous research, for in-stance in [7], [8]. An extensive amount of research about information and knowledge management in the context of PLM is also available, for instance [6], [9-11].

Tacit knowledge usually is subjective, cognitive and a result of experimental learning (knowledge that is still in the mind of the person) [6][12]. While tacit knowledge is formed into explicit, knowledge becomes more objective, rational and technical knowledge, (knowledge, that can be relatively easily saved, processed and shared, and that is well documented and accessible) [6], [12]. In this phase technological solutions have an active role [6].

The technological enablers for PLM are the different ICT tools used during the life of the product. Consequently, PLM can be described as multilayered and multisystem architecture [9]. From this ICT point-of-view, Garetti et al. [10] use 'connective tissue' to describe the role of PLM, for instance, the connection from design software to production and supply chain software in the extended enterprise [10]. The reason for this metaphor is that at the moment there is no comprehensive and well-accepted commercial PLM tool [9]. It is therefore appropriate to refer to PLM in ICT terms as a 'system of systems' [9]. Single systems are deficient without synthesis with other systems. In management sciences the system approach focuses on the whole, not on the separate parts, hence the relationships (interactions and compatibility) between the parts are important for the total sys-tem performance [13].

A philosophical view of PLM is to close the knowledge loops of the solutions enable creating, transforming and sharing the knowledge throughout the product lifecycle [6]. In order to close the knowledge loops, the interactions and compatibility of the IT solutions, integrations are needed. Integration of different software solutions such as CAD (Computer Aided Design), PDM (Product Data Management), ERP (Enterprise Resource Planning), and CRM (Customer Relationship Management) started some years ago [10]. The integration of the different software solutions is important in ensuring the information flow without any gaps between. However, less attention has been paid to the integration of operation and maintenance tools, i.e. the so-called asset management system.

Change management is also an important function of any PLM frame-work. There are systems that provide means for managing changes, but in practice there are still problems with assessing the scope of the change [14]. Changes, especially in complex engineering domains, can be 'initiated', such as front-end new requirements or new innovative solutions [15]. In addition, changes can be 'emergent', such as problems occurring in different lifecycle processes such as testing [15]. As observed by Eckert et al. [15], even if the changes can in both cases be handled the same way, their causes are different. It is important that changes, whatever their origin, are handled and managed systematically and effectively. Particular attention should be paid to change management while the product is in operations; during corrective or preventive maintenance interventions for instance. As highlighted by Ulrich [4], products frequently undergo changes such as upgrades, add-ons, adaptations, wear, flexibility in use and even consumption.

\section{Background - Use Case}

The case study for this research is, as mentioned, a large-scale and complex scientific facility: that of CERN located across the French-Swiss border, in the vicinity of 
Geneva, Switzerland. CERN is an international research center dedicated to experimental particle physics [16]. CERN equipment consists of accelerators, detectors and computing centers, which are substantially conceived in-house and partially manufactured, assembled and installed with the support of industrial firms.

As in many large-scale organizations, CERN has introduced and sometimes also developed different in-house means for keeping track of the equipment and their associated information. This need was enforced by the fact that several of CERN's facilities are subject to ionizing radiation and it is especially important to operate and maintain this equipment with a high degree of reliability and of traceability, and to accurately keep track of all pertinent information associated to equipment throughout its lifecycle.

The accelerator facility at CERN consists of many highly technological assets, but also of more common infrastructures such as industrial buildings and fully-equipped underground civil works. Technological assets include equipment from different decades. For instance, the PS mentioned above is from the 1950 s, while that of the Large Hadron Collider (LHC), a 27-km circumference superconducting accelerator, was built in the late 1990s and early 2000s, and commissioned in 2008.

The LHC relies on superconducting magnets (see figure 1), altogether 1232 main dipoles in two variants 1104 dipoles in the arcs and 128 dipoles in the so-called dispersion suppressor (DS) regions of the tunnel [17]. The LHC is not a perfect circle; it is made of eight arcs and of eight long straight sections (LSS) linked by16 DS sections [17]. Even if they count fewer components, LSS and DS are more demanding from an engineering point of view [17]. While the variants of the main cryodipole magnets share the same basic design, they may differ slightly in that they were manufactured by three different manufacturers [17].

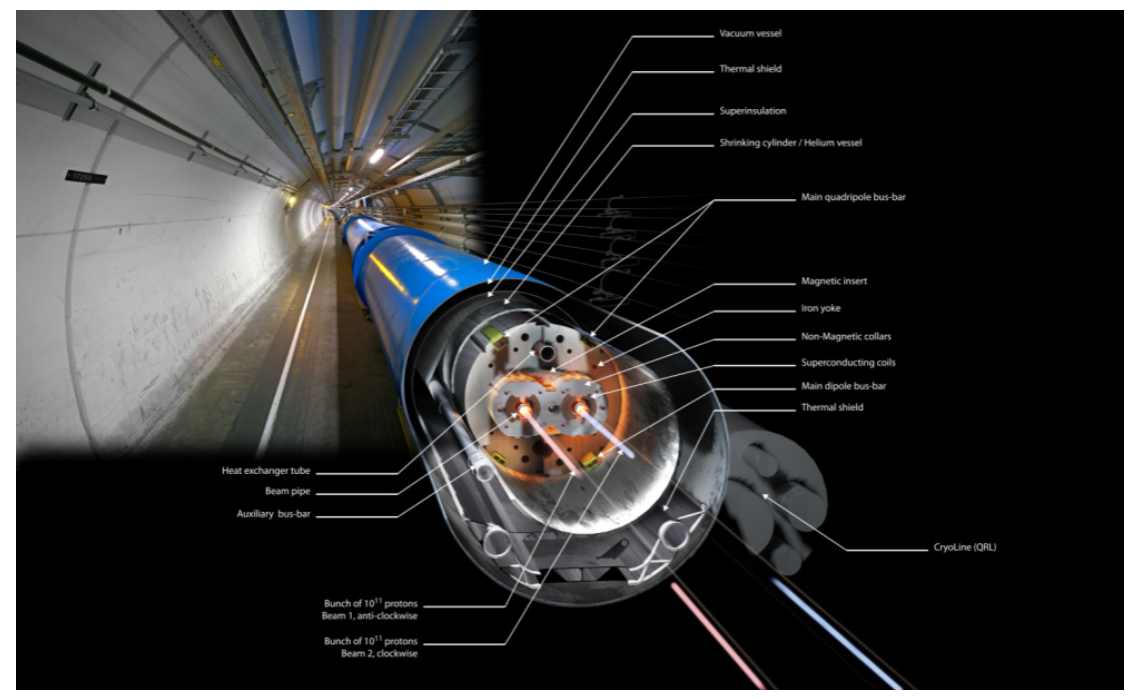

Fig. 1. Cross section of an LHC dipole in the tunnel. (C) 2011 CERN [18] 


\section{$4 \quad$ Items to Assets}

When equipment such as the superconducting cryodipole magnets in the LHC are designed, the CERN's design offices use the term 'item' to qualify and to identify the object being conceived. Items are in general any independent objects that have an identity [19]. Moreover, through items, for example products, product modules and components are identified, encoded and named in a systematic and standardized way [20].

At CERN, the LHC design information is managed through several systems: the still-in-use so-called CDD (CERN Drawing Directory, Oracle-base system developed for archiving 2D-CAD data), more recently CATIA ${ }^{\circledR}$ SmarTeam for handling 3D mock-ups and the CERN EDMS (Engineering \& Equipment Data Management Service). The typical characteristic of this early phase of the product lifecycle is that the objects are not yet tangible; they do not have yet a physical existence.

When design is ready and approved, the physical instances required are manufactured and assembled based on items. One item can lead to the manufacture and assembly of a one of a kind product. This is, for instance, the case of the LHC detectors, or in many instances the case of the 1232 units in two variants of the LHC cryodipole magnets described in section 3.

After their manufacture and assembly, either done in-house in one of CERN's many machine shops and workshops, or outsourced to specialized industrial firms through procurement contracts or to partner institutes through partnership agreements, the assets are finally installed and commissioned to achieve the function they were aimed at, the facilities can move to operations and maintenance. In the case of LHC cryodipole magnets, their function is the bending of the beams to keep the particles on an orbital trajectory.

Figure 2 describes the materialization of the intangible items used in the design of tangible assets used in operations and maintenance. This figure conveys the fact that conceptually our world is made of intangible objects, i.e. that do not have intrinsically a physical existence, and of tangible objects that have a physical existence. The model uses the well-know V-model (for example, [21], [22]) as a basis and places items and assets on the different branches of the V. Items can be broken down into sub-items and described accordingly along the descending left branch of the V-model; assets are integrated along the right branch of the V-model. In between is the materialization process. The materialization process aims to transform items into assets; out of one item, one to several assets can be materialized (the link 1 item to 1 ...n assets).

At CERN the LHC asset information is also managed through several systems mainly the CERN EDMS (Engineering \& Equipment Data Management Service) or the CERN CMMS (Computerized Maintenance Management System). This asset information can be stored as electronic files such as maintenance control records stored in the EDMS, or stored as data in the CMMS [23]. 


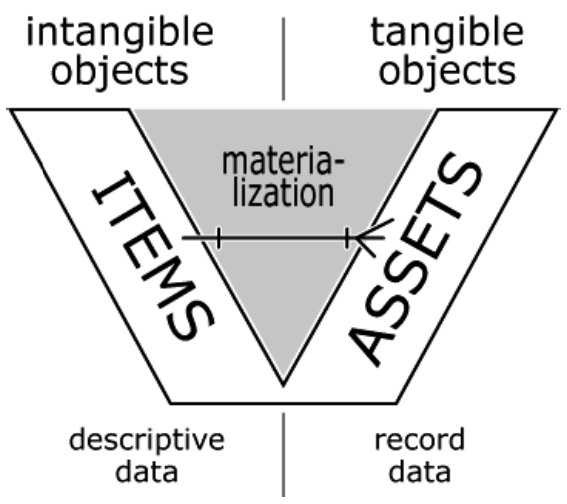

Fig. 2. V-model of materializing items to assets

As a general principle, equipment is manufactured and assembled based on engineering information developed at item level. However, a vast amount of information is also produced while operating and maintaining the equipment. If this information is generic, i.e. applicable to all instances of an asset, then it shall be at item level. If this information is specific to a given instance of equipment, then the asset shall be the repository for this information. Figure 3, adapted from basic quality management practices shows the categorization of different types of information. It is also of interest to notice that the information is different according to the phases of life of a product [23]. Information associated with an item shall typically be at 'descriptive level' i.e. level 3 in the quality assurance hierarchy of documents, while an asset's information shall be at 'records level' i.e. level 4 (see figures 2 and 3).

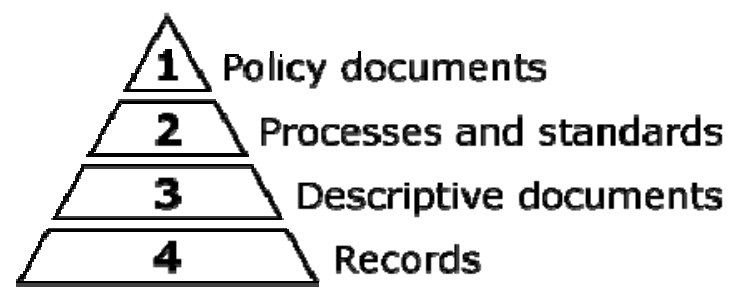

Fig. 3. Document hierarchies in CERN adapted from [23]

As described above, during the life of equipment at CERN there is a transfer from one terminology to another, but also transfer from one system of information systems (SmarTeam, CDD and EDMS) to another (EDMS and CMMS). The terms and systems mentioned here do not cover all of those in use at CERN, but are a simplification for the use in this paper. For instance following (sometimes synonymous) terms are in use: item, equipment, part, system, asset, functional position, slot and location. It becomes evident that if there is no link between the terminology and the information systems, there will be problems when the need to proceed to some modifications to the original design will appear. It is then of prime importance to keep the information coherent throughout the whole lifecycle of the equipment. 


\section{From Concepts to Practice}

When the items have been manufactured, installed and commissioned, the materialized equipment is handed over to the operation and maintenance phase. For the sake of operational efficiency, the CERN organizational structure is made of different sectors, departments groups and sections. For instance, the so-called Accelerator and Technology (A\&T) sector groups three departments, namely the Beams, Engineering and Technology Departments. Further down, the Engineering Department take care of particular part of the equipment, systems and facilities by means of specialized groups: e.g. Cooling and Ventilation Group, Electrical Engineering Group, Handling Engineering Group, Beam Instrumentation Group or Cryogenics Group to cite just a few. Finally through their sections, the groups provide services such as the operational maintenance of the equipment for which they have responsibility. The problem with such an organizational approach is that transverse processes such as maintenance operations, whether they are corrective or preventive, are performed in different departments and groups and rather independently.

In the handover process, an item is transformed into one or several assets. Figure 4 shows that after materialization items and assets may have independent lifecycles, but necessarily interdependent ones: design activities belong to the item lifecycle. Materialization is an activity that 'bridges' both lifecycle, i.e. that initiates an asset instance of an item. After materialization, an item in design may be subject to changes. There are many challenges in the change implementation, regardless of cause or type [24]. In the generic engineering change process introduced in Jarratt et al. [25], the first step is an engineering change request, which determines reason, priority and type of a change in addition to components and systems that are likely to be affected.

Changes to items, propagates to versioning or revising the item. Unfortunately, as pointed out in [26], the terminologies are not fixed. In any case, the meaning is that the item is changed in a matter that it will replace the original item [19], [26]. When changes are made as revisions, an important issue to keep in mind is the rules for revisions. The ISO 11442 standard dedicated to technical product documentation management [27] provides insights into revision rules for documents to reflect design changes. The revision rules also affect the identification of the components. The following passage of ISO 11442 [27] shall be kept in mind:

"Technical changes with requirement for interchangeability between the old and the new version. The form, fit and function of the component is not affected, and consequently the identification number is retained." [27]

This raises the question of interchangeability: while an item design evolves, how to guarantee that the results of its evolution are compatible with the requirements of its corresponding assets that are in use?

Sometimes items are changed in such a manner that this rule does not apply and the original item is not replaced. This process produces variants. Variants are parallel options for items [19], [26]. Items may evolve, while their corresponding assets are kept physically unchanged (see figure 4). A problem may arise when the need to 
replace or modify an asset appears. Shall the replaced or repaired asset be based on version 1 of the item (that may have an obsolete status) or on version 2 (that is the latest released version of the asset)? It is noteworthy that the figure 4 is meant to be a discussion opener for the topic.

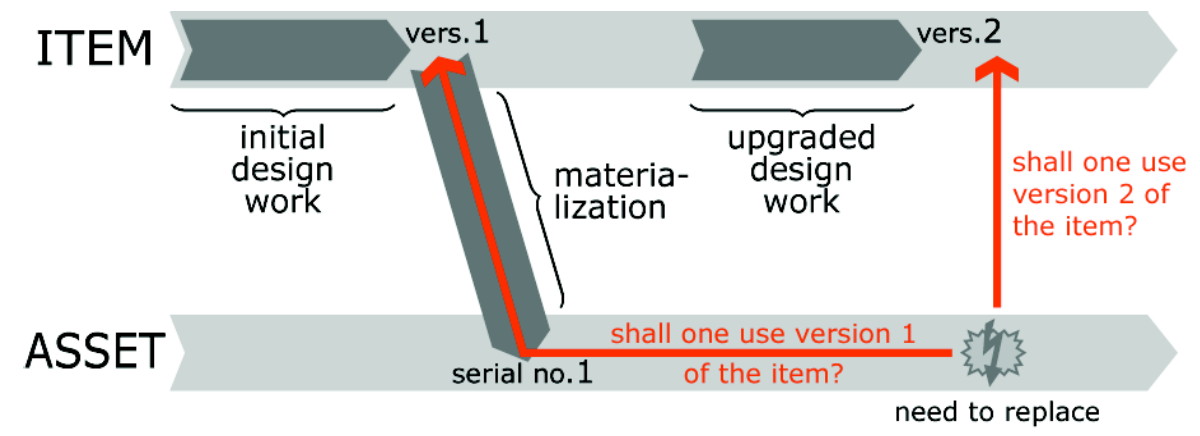

Fig. 4. Item and Asset Lifecycles

Another point of view on the information flow is backwards from assets to items. While modifying items the versioning and creation of variants are well defined procedures (e.g. [19], [26]), whereas describing what will happen in the item level when an asset is modified is to the best of our knowledge not well described. However, the modification of the description of an asset should be reflected at item level by creating a variant to receive this descriptive information.

\section{$6 \quad$ Summary and Conclusions}

In a complex and tailor-made environment, with the long life of equipment, it is extremely important to put emphasis on managing product information. The characteristics of the information such as that produced in different lifecycle phases and different period of time also bring their own flavour to the problems. In this paper we have raised and discussed a few issues: possible misunderstanding due to different

Table 1. The challenges discussed

\begin{tabular}{ll}
\hline Challenge & Description \\
\hline Concepts vs. Practice & $\begin{array}{l}\text { In system design objects are still intangible, while in operation and } \\
\text { maintenance the objects are tangible with a physical existence. In the } \\
\text { materialization process one intangible object is transformed into one or } \\
\text { several tangible objects and the link shall be maintained. }\end{array}$ \\
Terminology & $\begin{array}{l}\text { Different terminology is used in different lifecycle phases. Problems may } \\
\text { occur, when changing from term to term, and in the interfaces. }\end{array}$ \\
Different lifecycles & $\begin{array}{l}\text { Different objects (i.e. items and assets) may have different lifecycles. In } \\
\text { the need of modification or replacement there is a need for 'bridging' the } \\
\text { different lifecycles. }\end{array}$ \\
\hline
\end{tabular}


terminologies used over the lifecycle; and additional misunderstanding due an insufficient distinction between the key concepts of item and asset.

Throughout its whole lifecycle, a product is prone to change [25]. Engineering changes is a largely noticed issue in industry, but also an active research area of academia [25]. The paper also draws attention integrating the information from different lifecycle phases, from items to assets and vice versa, together.

Discussed important challenges are highlighted in table 1.

Acknowledgments. We would like to express our gratitude for European Commission Marie Curie Actions for providing funding for this research (FP7 grant agreement 264336). We would also like to thank PURESAFE-project consortium, especially CERN for their insight.

\section{References}

[1] European Commission, Directive 2006/42/EC of the European Parliament and of the Council of 17 May 2006 on machinery, and amending Directive 95/16/EC (2006)

[2] Technology monitoring 1102 Product Lifecycle Management, Berliner Kreis Wissenschaftliches Forum für Produktentwicklung e.V. WGP - Wissenschaftliche Gesellschaft für Produktionstechnik (September 2002), http://www.berlinerkreis.de, http: / /www.wgp.de

[3] ISO 10303, Industrial automation systems and integration - Product data representation and exchange (ISO 10303)

[4] Ulrich, K.: The role of product architecture in the manufacturing firm. Research Policy 24(3), 419-440 (1995)

[5] Maurer, M., Lindemann, U.: Individualized Product Design by Evolutionary Algorithms. In: Khosla, R., Howlett, R.J., Jain, L.C. (eds.) KES 2005. LNCS (LNAI), vol. 3683, pp. 1359-1365. Springer, Heidelberg (2005)

[6] Ameri, F., Dutta, D.: Product lifecycle management: Closing the knowledge loops. Computer-Aided Design and Applications 2(5), 577-590 (2005)

[7] Aamodt, A., Nygård, M.: Different roles and mutual dependencies of data, information, and knowledge - An AI perspective on their integration. Data \& Knowledge Engineering 16(3), 191-222 (1995)

[8] Tuomi, I.: Data is more than knowledge: implications of the reversed knowledge hierarchy for knowledge management and organizational memory. In: Proceedings of the 32nd Annual Hawaii International Conference on Systems Sciences, HICSS-32, Track1, 12. p (1999)

[9] Terzi, S., Bouras, A., Dutta, D., Garetti, M., Kiritsis, D.: Product lifecycle management from its history to its new role. International Journal of Product Lifecycle Management 4(4), 360-389 (2010)

[10] Garetti, M., Terzi, S., Bertacci, N., Brianza, M.: Organisational change and knowledge management in PLM implementation. International Journal of Product Lifecycle Management 1(1), 43-51 (2005)

[11] Abramovici, M.: Future Trends in Product Lifecycle Management (PLM). In: Krause, F.L. (ed.) The Future of Product Development, pp. 665-674. Springer, Heidelberg (2007)

[12] Gupta, B., Iyer, L.S., Aronson, J.E.: Knowledge management: practices and challenges. Industrial Management \& Data Systems 100(1), 17-21 (2000) 
[13] Ackoff, R.L.: Towards a System of Systems Concepts. Management Science 17(11), 661-671 (1971)

[14] Ahmad, N., Wynn, D.C., Clarkson, P.J.: Change impact on a product and its redesign process: a tool for knowledge capture and reuse. Res. Eng. Design, 1-26 (2012), doi:10.1007/s00163-012-0139-8

[15] Eckert, C., Clarkson, P.J., Zanker, W.: Change and customisation in complex engineering domains. Research in Engineering Design 15(1), 1-21 (2004)

[16] Research at CERN: a gateway to the Universe, http://public.web.cern.ch/ public/en/Research/Research-en.html (acessed: November 28, 2012)

[17] Evans, L., Bryant, P.: LHC Machine. Journal of Instrumentation 3(08), SO8001 (2008)

[18] Brice, M.: Cross section of an LHC dipole in the tunnel. CERN Document Server (July 07, 2011), http://cds.cern.ch/record/1365795 (accessed: February 02, 2013)

[19] Peltonen, H., Martio, A., Sulonen, R.: PDM: tuotetiedon hallinta. Edita. IT Press (2002)

[20] Sääksvuori, A., Immonen, A.: Product Lifecycle Management, 3rd edn. Springer, Berlin, Heidelberg (2008)

[21] Sage, A.P., Rouse, W.B.: Handbook of Systems Engineering and Management, 2nd edn. John Wiley \& Sons, Inc., Hoboken (2009)

[22] Hull, E., Jackson, K., Dick, J.: Requirements Engineering, 2nd edn. Springer (2004)

[23] Bonnal, P., Delamare, C., Mugnier, C., Pace, M., Perinic, G., Pilon, R., Rühl, I., Van Uytvink, E., Zaharieva, Z.: Maintenance Management Project Concept Report. CERN EDMS No. 1173942 (June 2012) (restricted Access)

[24] Clarkson, P.J., Simons, C.S., Eckert, C.M.: Predicting change propagation in complex design. In: Proceedings of the 13th International Conference on Design Theory and Methodology, Part of (DETC 2001) (September 12, 2001), http: / publications . eng.cam.ac.uk/328560/ (accessed: December 14, 2012)

[25] Jarratt, T.A.W., Eckert, C.M., Caldwell, N.H.M., Clarkson, P.J.: Engineering change: an overview and perspective on the literature. Res. Eng. Design 22(2), 103-124 (2011)

[26] Schichtel, M.: Produktdatenmodellierung in der Praxis. Carl Hanser Verlag, München (2002)

[27] ISO 11442, Technical product documentation - Document management (ISO 11442:2006) 